\title{
Electrically Switchable Entanglement Channel in van der Waals Magnets
}

\author{
H. Y. Yuan $\odot,{ }^{1, *}$ Akashdeep Kamra $\odot,{ }^{2,3}$ Dion M. F. Hartmann $\odot,{ }^{1}$ and Rembert A. Duine ${ }^{1,2}$ \\ ${ }^{1}$ Institute for Theoretical Physics, Utrecht University, Utrecht 3584 CC, Netherlands \\ ${ }^{2}$ Center for Quantum Spintronics, Department of Physics, Norwegian University of Science and Technology, \\ Trondheim 7491, Norway \\ ${ }^{3}$ Condensed Matter Physics Center (IFIMAC) and Departamento de Física Teórica de la Materia Condensada, \\ Universidad Autónoma de Madrid, Madrid 28049, Spain
}

(Received 5 April 2021; revised 15 June 2021; accepted 6 August 2021; published 26 August 2021)

\begin{abstract}
Two-dimensional layered van der Waals (vdW) magnets demonstrate their potential to allow the study of both fundamental and applied physics due to their remarkable electronic properties. However, the connection of $\mathrm{vdW}$ magnets to spintronics and quantum information science is not clear. In particular, it remains elusive whether there are interesting magnetic phenomena belonging only to vdW magnets but absent in widely studied crystalline magnets. Here, we consider the quantum correlations of magnons in a layered vdW magnet and identify an entanglement channel of magnons across the magnetic layers, which can be effectively tuned and even deterministically switched on and off by both magnetic and electric means. This is a unique feature of $\mathrm{vdW}$ magnets, in which the underlying physics is well understood in terms of the competing roles of exchange and anisotropy fields that contribute to magnon excitation. Furthermore, we show that such a tunable entanglement channel can mediate the electrically controllable entanglement of two distant qubits, which also provides a protocol to indirectly measure the entanglement of magnons. Our findings provide an avenue to electrically manipulate qubits and further open up opportunities to utilize vdW magnets for quantum information science.
\end{abstract}

DOI: 10.1103/PhysRevApplied.16.024047

\section{INTRODUCTION}

Two-dimensional (2D) van der Waals (vdW) magnets attract significant research interest for their remarkable features, which include rich physical phenomena in the $2 \mathrm{D}$ limit and desirable tunability of magnetic properties by the gate voltage [1-4]. Of particular interest is the interplay of $\mathrm{vdW}$ magnets with traditional spintronics, which manipulates spin transport and is controlled by a wide range of factors, including magnetization dynamics, magnetic field, microwave, charge current, thermal gradient, and mechanical strain. Even though spin-orbit-torque-induced magnetization switching $[5,6]$ and thermal-driven magnon transport in 2D magnets was recently demonstrated [7-10], resembling that observed in bulk magnets, it remains elusive whether there are interesting magnetic phenomena belonging only to the vdW magnets and whether they are

\footnotetext{
*huaiyangyuan@gmail.com
}

Published by the American Physical Society under the terms of the Creative Commons Attribution 4.0 International license. Further distribution of this work must maintain attribution to the author(s) and the published article's title, journal citation, and DOI. advantageous to advance the current horizon of spintronics. Here, we address this issue by considering the quantum correlations of magnons in a vdW magnet and its potential application in quantum information science. Primary opportunities for 2D materials have emerged in designing quantum-dot qubits, superconducting qubits, and topological quantum-computing platforms [11]. As we focus on 2D vdW magnets, however, the situation becomes different. Here, elementary excitation in the magnets is of magnons with a continuous spectrum instead of qubits with discrete energy levels, and thus, it becomes more natural for integration with continuous-variable quantum information [12], similar to the role of mechanical modes in optomechanics [13]. Generating and manipulating the quantum entanglement of continuous quantum variables is an essential step in this direction, and it also motivates our current work.

It is known that magnons excited from the classical ground state of crystalline ferrimagnets and antiferromagnets can form a squeezed state with finite entanglement at low temperature, and the strength of entanglement directly depends on the squeezing parameter [14-16]. However, this entanglement is not tunable by external fields as long as the spins are antiparallel to each other. To overcome this bottleneck, cavity photons can be used to enhance 
magnon-magnon entanglement near the resonance [14]. Alternatively, one may achieve a distant and tunable magnon-magnon entanglement by coupling two magnets simultaneously with one cavity field $[17,18]$.

Here, we study the entanglement of magnons in a layered vdW magnet and identify an entanglement channel that is robust against magnetic dissipation. Magnon entanglement through this channel can be effectively tuned by both magnetic and electric fields. It is a unique feature of vdW magnets with comparable strength of exchange and anisotropy fields and is absent in most bulk magnets. In particular, a nonmonotonic dependence of magnonmagnon entanglement on the two-mode squeezing parameter is identified, which can only be fully understood by including the competing roles of single-mode squeezing, two-mode squeezing, and two-mode coherent conversion. We further show that such an entanglement channel can bridge the entanglement of two qubits, and it can be electrically switchable by tuning the gate voltage. Our results may significantly extend the research horizon of $\mathrm{vdW}$ magnets and promote the application of $\mathrm{vdW}$ magnets in quantum information science.

\section{PHYSICAL MODEL}

Let us consider the layered vdW magnet shown in Fig. 1(a), which is described by the following Hamiltonian:

$$
\mathcal{H}=\sum_{i j, \mathbf{R}, \mathbf{R}^{\prime}} J_{i j} \mathbf{S}_{i, \mathbf{R}} \cdot \mathbf{S}_{j} \mathbf{R}^{\prime}-K \sum_{i, \mathbf{R}}\left(S_{i, \mathbf{R}}^{z}\right)^{2}-\mathbf{H} \cdot \sum_{i, \mathbf{R}} \mathbf{S}_{i, \mathbf{R}},
$$

where $i, j \in\{1,2\}$ are layer indices; $\mathbf{R}$ is the position of magnetic atoms; and the diagonal and off-diagonal elements of exchange-matrix $J_{i j}$ represent intralayer and interlayer nearest-neighbor exchange coupling, respectively. Furthermore, $K>0$ is the anisotropy coefficient, and $\mathbf{H}$ is the applied magnetic field. The strengths of both exchange and anisotropy fields are tunable by modifying the density of states of electrons through electric fields. Since the intralayer exchange coupling is much larger than the interlayer coupling $\left(J_{12} \ll J_{11}, J_{22}\right)$, the magnetization in each layer usually behaves like a macrospin. Following the experimental setup realized for $\mathrm{CrI}_{3}$ [19], we consider an in-plane field, $\mathbf{H}=H e_{y}$. Then the classical ground state of the system is a canted state, $\theta=$ $\arcsin H / H_{c m}$, when $H<H_{c m}$, and a collinear state, $\theta=$ $\pi / 2$, when $H \geq H_{c m}$, where the magnetic transition field is $H_{c m} \equiv 2(J+K) S$ with $J_{12}=J_{21} \equiv J / 2$ [20]. To study the magnons in this ground state, we first rotate to a new frame $\left(x^{\prime}, y^{\prime}, z^{\prime}\right)$, with $z^{\prime}$ pointing along the equilibrium orientation of magnetization and $x^{\prime}=x$, and perform a Holstein-Primakoff transformation [21] near the classical ground state, i.e., $S_{1, \mathbf{R}}^{+}=\sqrt{2 S} a_{1, \mathbf{R}}, S_{1, \mathbf{R}}^{-}=\sqrt{2 S} a_{1, \mathbf{R}}^{\dagger}, S_{1, \mathbf{R}}^{z^{\prime}}=$ $S-a_{1, \mathbf{R}}^{\dagger} a_{1, \mathbf{R}}$, where $S_{1, \mathbf{R}}^{+}\left(S_{1, \mathbf{R}}^{-}\right)$are spin-rising (-lowering) (a)

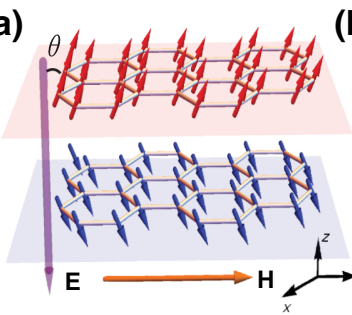

vdW magnet

(c)

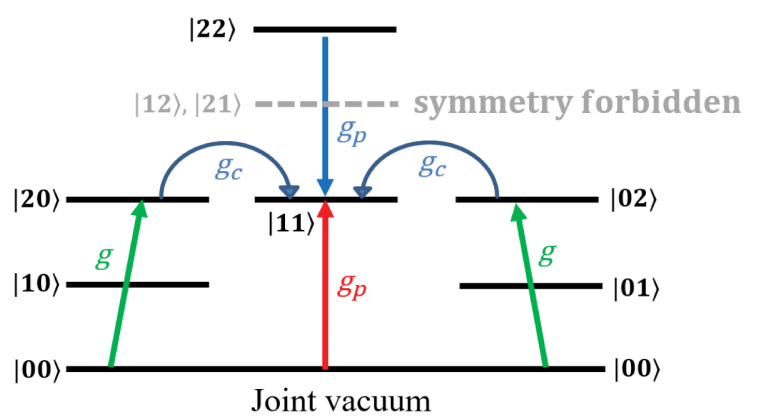

FIG. 1. (a) Schematic illustration of bilayer van der Waals magnets subject to an in-plane magnetic field $(\mathbf{H})$ and a normal electric field (E) (b) Entanglement of magnons and occupation of Fock space in the ground state as a function of magnetic field. Magnetic parameters of $\mathrm{CrI}_{3}$ [19] are used with $2 J=0.83 \mathrm{~T}$ and $2 K=1.73 \mathrm{~T}$. We take the truncation $N=19$ in the numerical calculation of magnon entanglement (red line) and a further increase of $N$ does not change the entanglement significantly. Red dashed line indicates the magnetic transition field at $H_{c m}=$ $2.46 \mathrm{~T}$, and blue dashed line is the local minimum of entanglement at $H_{c e}=2.16 \mathrm{~T}$. (c) Energy levels of the two-magnon system.

operators and $a_{1, \mathbf{R}}\left(a_{1, \mathbf{R}}^{\dagger}\right)$ are the magnon-annihilation (-creation) operators at position $\mathbf{R}$ of the first magnetic layer, which satisfy the commutation relations $\left[a_{1, \mathbf{R}}, a_{1, \mathbf{R}^{\prime}}^{\dagger}\right]=\delta_{\mathbf{R R}^{\prime}}$. Similar operations hold for the spins in the second layer. By rotating back to the old frame $(x, y, z)$, substituting the spin operators into the Hamiltonian [Eq. (1)] and further making a Fourier transform, $a_{j, \mathbf{R}}=1 / \sqrt{N_{s}} \sum a_{j, \mathbf{k}} e^{i \mathbf{k} \cdot \mathbf{R}}$, with $N_{s}$ being the total number of spins, the effective Hamiltonian can be written as

$$
\begin{aligned}
\mathcal{H}= & \sum_{i=1}^{2} \omega_{0} a_{i}^{\dagger} a_{i}+\frac{1}{2} g\left(a_{i}^{\dagger} a_{i}^{\dagger}+a_{i} a_{i}\right) \\
& +g_{c}\left(a_{1}^{\dagger} a_{2}+a_{1} a_{2}^{\dagger}\right)+g_{p}\left(a_{1}^{\dagger} a_{2}^{\dagger}+a_{1} a_{2}\right)
\end{aligned}
$$

where $\omega_{0}=(J+K) S \cos 2 \theta+K S \cos ^{2} \theta+H \sin \theta, g=$ $K S \sin ^{2} \theta, g_{p}=J S \cos ^{2} \theta, g_{c}=J S \sin ^{2} \theta$, and $a_{i} \equiv a_{i, \mathbf{k} \rightarrow 0}$. We take the long-wavelength limit $(|\mathbf{k}| \rightarrow 0)$ because we focus on the low-energy magnons for simplicity. The firstorder terms, $a_{i}$ and $a_{i}^{\dagger}$, disappear once we take into account the ground-state configurations. 


\section{GROUND STATE}

Let us first study the ground-state properties, which will be useful for understanding the quantum correlations of magnons subject to dissipation. By numerically solving the Schrödinger equation, $\mathcal{H}|\psi\rangle=E|\psi\rangle$, in the bipartite Fock basis, where $|\psi\rangle=\sum_{m, n=0}^{N} C_{m n}|m n\rangle$, and $|m n\rangle$ refers to the occupation of the Fock state with particle numbers $m$ and $n$ in layers 1 and 2, respectively, we can derive the energy and wave function of the ground state, after taking a proper truncation of the maximum number of magnon excitations $(N)$. In general, one may quantify the strength of entanglement using the von Neumann entropy, Duan-Simon criteria [22,23], and logarithmic negativity $[24,25]$. To be consistent with the discussion on the mixed-state case presented below, we choose logarithmic negativity, where a state with zero logarithmic negativity is separable and that with larger logarithmic negativity has stronger entanglement. To calculate logarithmic negativity in the magnonic systems, we first derive the covariance matrix (CM) of the system in the form $\mathbf{V}_{l l^{\prime}}=\left(\left\langle u_{l} u_{l^{\prime}}\right\rangle+\right.$ $\left.\left\langle u_{l^{\prime}} u_{l}\right\rangle\right) / 2$, where $\mathbf{u}=\left(x_{1}, p_{1}, x_{2}, p_{2}\right),\left\langle u_{l} u_{l^{\prime}}\right\rangle=\left\langle\psi\left|u_{i} u_{j}\right| \psi\right\rangle$, and the quadrature operators are defined as $x_{i}=\left(a_{i}+\right.$ $\left.a_{i}^{\dagger}\right) / \sqrt{2}$ and $p_{i}=-i\left(a_{i}-a_{i}^{\dagger}\right) / \sqrt{2}$. Due to the permutation symmetry of the system $(1 \leftrightarrow 2)$, the $\mathrm{CM}$ should take the form $\mathbf{V}_{4 \times 4}=\left(\begin{array}{ll}\mathbf{A}_{2 \times 2} & \mathbf{C}_{2 \times 2} \\ \mathbf{C}_{2 \times 2}^{T} & \mathbf{A}_{2 \times 2}\end{array}\right)$, then the logarithmic negativity is calculated as $E_{N}=\max \left[0,-\ln \left(2 \eta^{-}\right)\right]$, where $\eta^{-}=\sqrt{\sum \mathbf{V}-\sqrt{\sum \mathbf{V}^{2}-4 \operatorname{det} \mathbf{V}}}$ with $\sum \mathbf{V}=$ $2 \operatorname{det}(\mathbf{A})-2 \operatorname{det}(\mathbf{C})$ and $\operatorname{det}(\mathbf{V})$ as the two symplectic invariants of $\mathbf{V}$ [24].

Figure 1(b) shows that, with an increasing magnetic field, the entanglement first drops until a field named as $H_{c e}$ before the magnetic transition and then rises to a peak at the transition $\left(H_{c m}\right)$ (red dashed line). For comparison, the two-mode squeezing, $\Delta x=\sqrt{\left\langle\left(x_{1}+x_{2}\right)^{2}\right\rangle}$, takes on a single minimum at $H_{c m}$ (green line). In the case of the wave function being described by a two-mode squeezed state, the entanglement becomes a monotonous function of the squeezing parameter $[14,15,24]$. In contrast, our system deviates from such a wave function, and thus, admits additional entanglement channels, as discussed further below.

To understand the essential physics, we first analyze the symmetry of the Hamiltonian. Both on-site squeezing, $a_{i}^{\dagger} a_{i}^{\dagger}$, and intersite squeezing, $a_{1}^{\dagger} a_{2}^{\dagger}$, allow only an even number of excitations $(|00\rangle,|02\rangle,|11\rangle,|20\rangle, \ldots)$, such that the ground-state wave function can be written as $|\psi\rangle$ $=C_{0}|00\rangle+C_{2}|02\rangle C_{2}+C_{3}|11\rangle+C_{2}|20\rangle+C_{4}|22\rangle[26]$, where we truncate the Hilbert space at $N=2$. As we shall see, this is sufficient to capture the qualitative physics. The entanglement of such a state means that it cannot be factorized as $|\psi\rangle=\left|\psi_{1}\right\rangle \otimes\left|\psi_{2}\right\rangle$, where $\left|\psi_{i}\right\rangle=c_{0}|0\rangle+$ $c_{1}|1\rangle+c_{2}|2\rangle$. This factorization is straightforward when $c_{1}=0$. Given $c_{1} \neq 0$, this factorization is impossible for the inevitable appearance of odd excitation $(|10\rangle,|01\rangle \cdots)$. Hence, it is expected that the occupation of $|11\rangle\left(c_{1}\right)$ is crucial for determining the entanglement of the ground state. On the other hand, as shown in Fig. 1(c), the occupation of $|11\rangle$ acquires contributions from two competing effects: (i) the direct parametric excitation $\left(g_{p}\right)$, the strength of which decreases monotonically as $\theta$ increases from zero to $\pi / 2$; and (ii) the coherent transfer of $|11\rangle$ to $|02\rangle$ and $|20\rangle\left(g_{c}\right)$ followed by an on-site parametric process to $|00\rangle(g)$ and the reversal process, the strength of which increases as $\theta$ increases. These two channels interfere destructively and result in a minimal entanglement state at a critical value of $H_{c e}$ before $H_{c m}$. Above $H_{c e}$, the coherent channel dominates the entanglement, and it keeps increasing up to the magnetic transition field, $H_{c m}$. As we increase the fields further, $g_{c}$ does not change any more, while the gap between $|00\rangle$ and $|02\rangle$ keeps increasing to suppress on-site parametric excitation. Hence, the occupation of $|11\rangle$ and the ground-state entanglement decrease.

Analytically, we can solve the Schrödinger equation, $\mathcal{H}|\psi\rangle=E|\psi\rangle$, to derive the superposition coefficients as

$$
\begin{aligned}
& C_{0} \approx 1, C_{01}=C_{10}=C_{12}=C_{21}=0, \\
& C_{2}=\frac{\sqrt{2}}{2} \frac{g \epsilon^{2}+2\left(g_{c} g_{p}-6 g \omega_{0}\right) \epsilon-2 g g_{p}^{2}-8 g_{c} g_{p} \omega_{0}+8 g \omega_{0}^{2}}{\epsilon^{3}-8 \omega_{0}^{2} \epsilon^{2}-\left[g^{2}+4\left(g_{c}^{2}+g_{p}^{2}-5 \omega_{0}^{2}\right)\right] \epsilon+8 \omega_{0}\left(2 g_{c}^{2}+g_{p}^{2}-2 \omega_{0}^{2}\right)+2 g^{2} \omega_{0}^{2}-8 g g_{c} g_{p}}, \\
& C_{3}=\frac{g_{p} \epsilon^{2}+2\left(g g_{c}-3 g_{p} \omega_{0}\right) \epsilon+g^{2} g_{p}-8 g g_{c} \omega_{0}+8 g_{p} \omega_{0}^{2}}{\epsilon^{3}-8 \omega_{0}^{2} \epsilon^{2}-\left[g^{2}+4\left(g_{c}^{2}+g_{p}^{2}-5 \omega_{0}^{2}\right)\right] \epsilon+8 \omega_{0}\left(2 g_{c}^{2}+g_{p}^{2}-2 \omega_{0}^{2}\right)+2 g^{2} \omega_{0}^{2}-8 g g_{c} g_{p}}, \\
& C_{5}=\frac{\left(g^{2}+2 g_{p}^{2}\right) \epsilon+6 g g_{c} g_{p}-2 g^{2} \omega_{0}-4 g_{p}^{2} \omega_{0}}{\epsilon^{3}-8 \omega_{0}^{2} \epsilon^{2}-\left[g^{2}+4\left(g_{c}^{2}+g_{p}^{2}-5 \omega_{0}^{2}\right)\right] \epsilon+8 \omega_{0}\left(2 g_{c}^{2}+g_{p}^{2}-2 \omega_{0}^{2}\right)+2 g^{2} \omega_{0}^{2}-8 g g_{c} g_{p}},
\end{aligned}
$$


where $\epsilon$ is the ground-state energy and is equal to the minimum root of the following quartic equation:

$$
\begin{aligned}
x^{4} & -8 \omega_{0} x^{3}-2\left(g^{2}+2 g_{c}^{2}-10 \omega_{0}^{2}\right) x^{2} \\
& +2\left(2 g g_{c} g_{p}+g^{2} g_{p}-4 g^{2} \omega_{0}-8 g_{c}^{2} \omega_{0}+8 \omega_{0}^{3}\right) x \\
& +4 g\left[g\left(g_{p}-2 \omega_{0}\right) \omega_{0}-2 g_{c} g_{p}^{2}\right]=0 .
\end{aligned}
$$

The solid lines in Fig. 1(b) depict this analytical calculation of particle occupation, and it shows that minimum entanglement indeed happens when the occupation of $|11\rangle$ is minimum, which is consistent with the intuitive picture.

\section{ROLE OF DISSIPATION}

A real van der Waals magnet is far from isolated because it is inevitably in contact with the environment, such as a phonon bath. Thus, the quantum states of magnons may suffer from dissipation due to, for example, the spinphonon interaction. The dissipation effect will mix the contributions from ground and excited states and stabilize the system in a steady state. In general, the steady state described by a density matrix can be characterized by its first and second moments. To calculate these two moments, we follow the standard fluctuation-dissipation theorem to introduce a noise term, $\sqrt{2 \gamma_{i}} \zeta_{i}(t)$, together with a decay term, $-\gamma a_{i}$, of the magnon mode into the Heisenberg equation of the system. Here, $\gamma_{1}$ and $\gamma_{2}$ are the dissipation coefficients of the two magnetic layers, and the fluctuation terms obey the statistics $\left\langle\zeta_{i}(t)\right\rangle=0$ and $\left\langle\zeta_{i}(t) \zeta_{j}^{\dagger}\left(t^{\prime}\right)\right\rangle=$ $\left(n_{\text {th }}+1\right) \delta_{i j} \delta\left(t-t^{\prime}\right)$, where $n_{\text {th }}$ is the thermal occupation of magnons. For the lack of coherent pumping in Eq. (2), $\left\langle a_{i}\right\rangle=\left\langle\zeta_{i}(t)\right\rangle=0$. The second moments, $\left\langle a_{i} a_{j}\right\rangle$, can be calculated by solving the Lyapunov equation, MV + $\mathbf{V M}=-\mathbf{D}$ [27]. Here, $\mathbf{D}=\operatorname{diag}\left[\left(2 n_{\text {th }}+1\right) \gamma_{1},\left(2 n_{\text {th }}+\right.\right.$ 1) $\left.\gamma_{1},\left(2 n_{\mathrm{th}}+1\right) \gamma_{2},\left(2 n_{\mathrm{th}}+1\right) \gamma_{2}\right)$, and the drift matrix of the system $(d \mathbf{u} / d t=\mathbf{M} \cdot \mathbf{u}]$ is

$$
\mathbf{M}=\left(\begin{array}{cccc}
-\gamma_{1} & -g+\omega_{0} & 0 & g_{c}-g_{p} \\
-g-\omega_{0} & -\gamma_{1} & -g_{c}-g_{p} & 0 \\
0 & g_{c}-g_{p} & -\gamma_{2} & -g+\omega_{0} \\
-g_{c}-g_{p} & 0 & -g-\omega_{0} & -\gamma_{2}
\end{array}\right) .
$$

Then, we can again quantify the entanglement by calculating the logarithmic negativity. In principle, this approach involves the contribution of all excited states in the Hilbert space, and hence, there are no truncation problems in solving the ground states, and it is safe to use logarithmic negativity to quantify the strength of entanglement. Figure 2(a) shows magnon-magnon entanglement in the plane of $(H, J / K)$. Depending on the values of $J / K$, two regimes can be classified, as shown in Fig. 2(b), which are extracted from the phase diagram for $J / K=0.48$ (red line), 0.96 (blue line), and 1.92 (black line). For $J / K<$

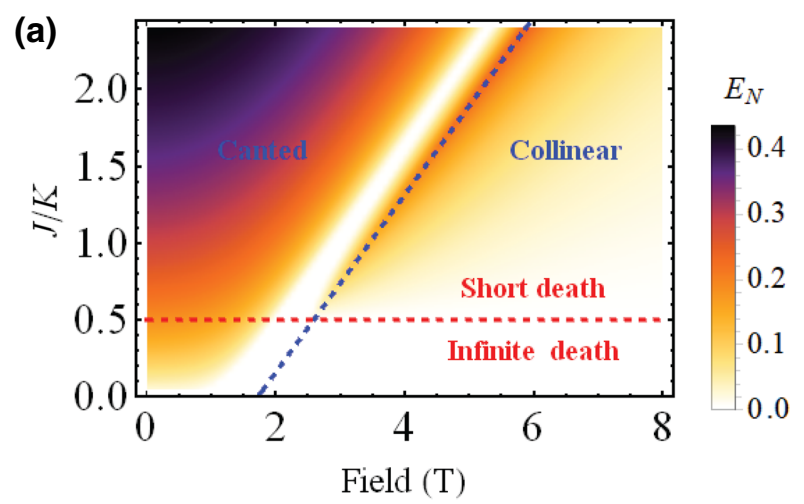

(b)

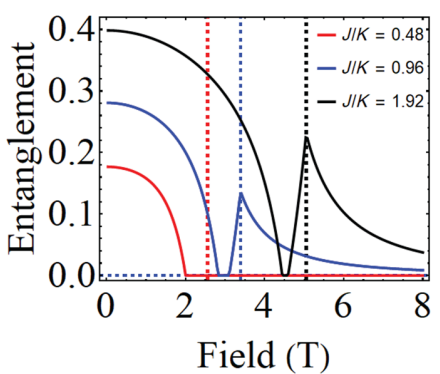

(c)

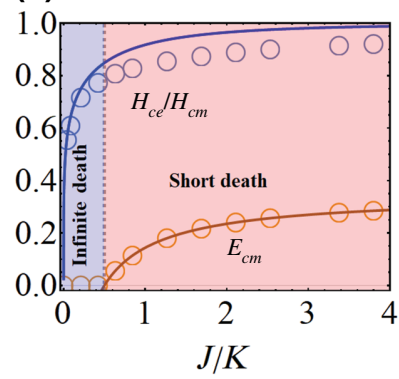

FIG. 2. (a) Steady magnon-magnon entanglement in the $(H, J / K)$ plane. (b) Steady entanglement as a function of external field for $J / K=0.48$ (red line), 0.96 (blue line), and 1.92 (black line). Dashed lines indicate the transition fields $H_{c m}$ located at $2(J+K) S$. $\gamma_{1}=\gamma_{2}=0.01$. (c) Entanglement at the transition field $\left(E_{c m}\right)$ and critical field for zero entanglement $\left(H_{c e} / H_{c m}\right)$ as a function of $J / K$. Symbols are full numerical results based on the Hamiltonian [Eq. (2)] and lines depict Eqs. (9) and (11).

0.5 , the entanglement monotonically decreases to zero at a critical field $H_{c e}<H_{c m}$ and is never revived again, while, for $J / K>0.5$, the entanglement first dies at $H_{c e}$, is revived after a finite-field range, and further reaches a local maximum at transition field $H_{c m}$. Such a field dependence resembles the ground-state case, as discussed for Fig. 1. This similarity suggests that the coherent channel and parametric channel still manifest themselves in the steady state. However, the coherent channel is more easily switched off under the influence of dissipation at smaller $J$. To illustrate this point, we discuss these regimes in detail as follows.

Let us first study the low-field regime by setting $g_{c}=0$. Then, we can analytically derive the CM and calculate the magnon-magnon entanglement as

$E_{N}=\max \left\{0, \frac{1}{2} \ln \frac{\left[\left(\omega_{0}+g_{p}\right)^{2}-g^{2}\right]\left[\left(\omega_{0}-g_{p}\right)^{2}-g^{2}\right]}{\left(\omega_{0}^{2}-g_{p} \sqrt{\omega_{0}^{2}+\gamma^{2}}\right)^{2}-g^{2}\left(\omega_{0}^{2}+\gamma^{2}\right)}\right\}$. 
Since dissipation is usually sufficiently smaller than the onsite frequency $\left(\gamma_{1}=\gamma_{2} \equiv \gamma \ll \omega_{0}\right)$ [28], $E_{N}$ can be further simplified as

$$
E_{N}=\max \left[0, \ln \sqrt{\left(\omega_{0}+g_{p}\right)^{2}-g^{2}}-\ln \omega_{0}\right] .
$$

One immediately sees that the entanglement positively depends on the parametric excitation of two types of magnons $\left(g_{p}\right)$, and there is a critical condition at which the steady entanglement is zero, i.e.,

$$
g_{p}^{2}+2 \omega_{0} g_{p}-g^{2}=0
$$

After substituting the field dependence of $g, g_{p}, \omega_{0}$, we can calculate the critical magnetic field by

$$
H_{c e}=H_{c m} \sqrt{\frac{3 J / K+4}{2 J / K+3+\sqrt{J^{2} / K^{2}+2 J / K+4+4 K / J}}} .
$$

It is straightforward to verify that $H_{c e}$ is a monotonically increasing function of $J / K$, and it approaches zero when $J / K \rightarrow 0$ and $H_{c m}$ when $J / K \rightarrow \infty$. This prediction describes the numerical results quite well for small $J / K$, as shown in Fig. 2(c). As $J / K$ increases, the critical field approaches $H_{c m}$, and the influence of the coherent process needs to be included.

In the coherent regime $\left(g_{p}=0\right)$, the $\mathrm{CM}$ of the system is also analytically solvable, and the logarithmic negativity is

$$
E_{N}=\max \left[0, \frac{1}{2} \ln \frac{\omega_{0}^{2}-\left(g-g_{c}\right)^{2}}{\omega_{0}^{2}-g_{c}^{2}}\right]
$$

This result implies that magnons are not entangled for $g>$ $2 g_{c}$ (or equivalently $K>2 J$ ), which can be easily satisfied in vdW magnets but is not favorable in crystalline magnets $(J \gg K)$. This readily explains the infinite death of entanglement without revival observed in Fig. 2(a). Here, the tunability of $J / K$ through the electric field enables an electrically switchable entanglement channel, as we shall see below. In the regime $g<2 g_{c}(K<2 J)$, there will be a finite entanglement between magnons near the magnetic transition field. Depending on the magnitude of the applied field, two regimes can be classified. (i) When $H \geq H_{c m}$, $\theta=\pi / 2$,

$$
E_{N}^{>}=-\frac{1}{2} \ln \left[1-\frac{(2 J-K) K S^{2}}{(H-2 K S)(H-2 J S)}\right],
$$

which decreases monotonically as the field increases, and the maximum entanglement at $H=H_{c m}$ is $E_{c m}=$ $\ln (\sqrt{1+K / 2 J} / \sqrt{2})$. (ii) When $H<H_{c m}, \theta=\arcsin H /$ $H_{c m}$, the logarithmic negativity can be rewritten as

$$
E_{N}^{<}=\sqrt{\frac{a_{4}^{\prime} H^{4}+a_{2} H^{2}+a_{0}}{a_{4} H^{4}+a_{2} H^{2}+a_{0}}},
$$

where $a_{4}^{\prime}=(J+K)(J-K), \quad a_{4}=J(J-2 K), \quad a_{2}=$ $8 K S^{2}(J+2 K)(J+K)^{2}$, and $a_{0}=-16 S^{4}(J+2 K)^{2}(J+$ $K)^{4}$. Notably, $a_{4}^{\prime}>a_{4}$ due to $K<2 J$; this implies that the entanglement will increase with the external field and reach its maximum value, $E_{c m}$, at $H_{c m}$. The prediction of this maximum entanglement captures the numerical result perfectly, as shown in Fig. 2(c) (brown line).

Until now, we have considered the case with zero thermal occupation of magnons, i.e., $T=0$. In reality, thermal fluctuations may induce the decoherence of magnons, and thus, weaken magnon-magnon entanglement. Figure 3(a) shows that the entanglement is still switchable up to thermal occupation $n_{\text {th }}=0.3$, which corresponds to a temperature of $T=1.6 \mathrm{~K}$. This temperature is well below the Curie temperature of $\mathrm{CrI}_{3}(\sim 45 \mathrm{~K}$ [2]) and is reasonable to realize using mature cryogenic technology. Such a stability range of entanglement is larger than the reported value below $0.2 \mathrm{~K}$ in a cavity containing two magnets [17]. On the other hand, the entanglement properties are insensitive to the dissipation of magnons, as shown in Fig. 3(b), which is consistent with the analytical result, Eq. (6), obtained at zero temperature, and similar results are also found in a collinear magnet [14]. Such insensitivity may be an advantage of magnonic systems compared with optomechanical systems, where the entanglement sensitively depends on dissipation [29]. The underlying physics of this difference requires further investigation. (a)

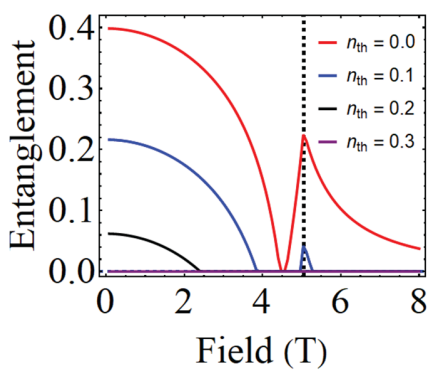

(b)

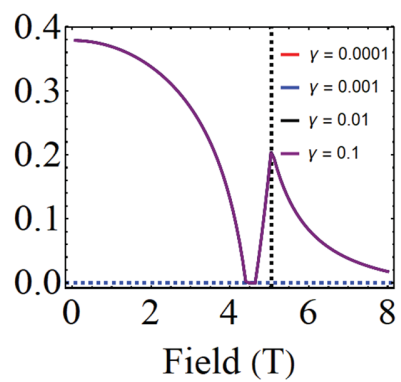

FIG. 3. Entanglement of magnons at different temperatures (a) and magnon dissipation (b). Here, $n_{\mathrm{th}}=0.3$ corresponds to $T=$ $1.6 \mathrm{~K}$ with $\omega / 2 \pi=50 \mathrm{GHz}$ [19]. $\gamma=0.01$ for (a) and $n_{\mathrm{th}}=$ 0.01 for (b). 


\section{QUBIT-QUBIT ENTANGLEMENT}

We demonstrate a tunable and robust entanglement channel in bilayer vdW magnets by applying a magnetic field. Furthermore, this quantum channel can also be turned on and off by an electric field through the modification of exchange and anisotropy fields. With bilayer $\mathrm{CrI}_{3}$ as an example, recent experiments show that both the exchange and anisotropy fields decrease with the gate voltage, $V$, by $J=J_{0}-b_{J} V$ and $K=K_{0}-b_{K} V$, where the coefficients $J_{0}, b_{J}, K_{0}$, and $b_{K}$ are extracted from previous experiments [19]. By taking this dependence into account, we numerically calculate the two-magnon entanglement as a function of gate voltage at different values of magnetic fields in Fig. 4(a) (solid lines). Clearly, the gate voltage can be an effective factor to switch the entanglement of magnons. This channel is robust, even if the exchange and anisotropy fields deviate a little from the experimental values we use, as long as their strengths are comparable. In typical crystalline antiferromagnets, anisotropy variation

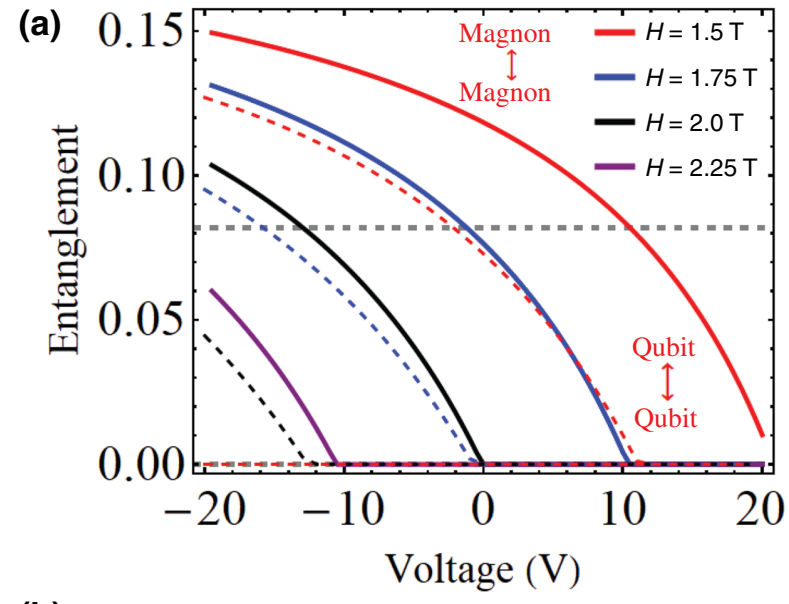

(b)
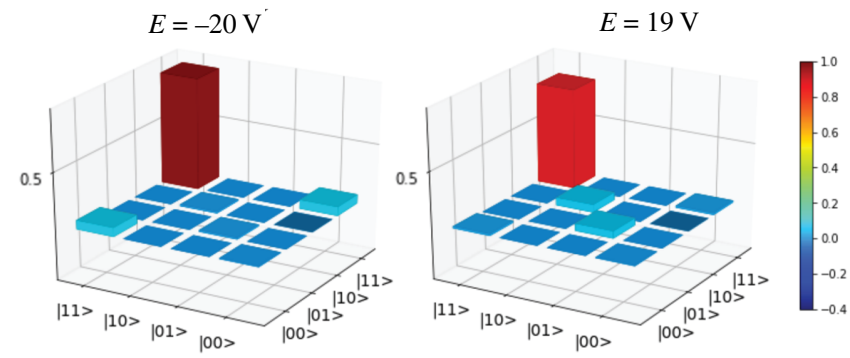

FIG. 4. Entanglement of magnons (solid lines) and qubits (dashed lines) as a function of gate voltage for $H=1.5 \mathrm{~T}$ (red line), $1.75 \mathrm{~T}$ (blue line), 2.0 T (black line), and 2.25 T (purple line). Gray line at $E_{N}=0.085$ is a threshold of magnon-magnon entanglement to switch the qubit-qubit entanglement on and off. Parameters are $J_{0}=0.865 \mathrm{~T}, b_{J}=9.5 \times 10^{-3} \mathrm{~T} / \mathrm{V}, K_{0}=$ $0.415 \mathrm{~T}$, and $b_{K}=5.5 \times 10^{-3} \mathrm{~T} / \mathrm{V}$ [19]. $\omega_{1}=-\omega_{2}=0.01 \mathrm{~T}$, $g_{q m}=10^{-4} \mathrm{~T}[30,31]$. (b) Density of states for the steady states of two qubits when $E=-20 \mathrm{~V}$ (left panel) and $19 \mathrm{~V}$ (right panel). $H=1.5 \mathrm{~T}$. caused by the electric field will not have a significant influence on the entanglement channel, because the magnitude of anisotropy is usually 3 orders of magnitude smaller than the exchange field.

This tunable entanglement provides an avenue to manipulate two qubits electrically. As a proof of concept, we consider two qubits coupled with the two layers of the $\mathrm{vdW}$ magnets through dipolar interactions. Under the rotatingwave approximation, the total Hamiltonian is written as [32]

$$
\mathcal{H}_{t}=\mathcal{H}+\sum_{i=1}^{2} \omega_{i} \sigma_{i}^{z}+g_{q m} \sum_{i=1}^{2}\left(\sigma_{i}^{+} a_{i}+\sigma_{i}^{-} a_{i}^{\dagger}\right)
$$

where $\sigma_{i}^{z}, \sigma_{i}^{ \pm}$are the Pauli matrices describing the $i$ th qubit, $\omega_{i}$ is the resonance frequency of the qubit, and $g_{q m}$ is the coupling strength. Here, we first solve the steadydensity matrix of the whole system, $\rho$, numerically and then calculate the reduced density matrix of the two qubits by tracing out the magnon degree of freedom, i.e., $\rho_{12}=$ $\operatorname{tr}_{a_{1} a_{2}}(\rho)$. Then we can quantify qubit-qubit entanglement by concurrence, which is defined as $C_{12}=\max \left(0, \lambda_{1}-\right.$ $\lambda_{2}-\lambda_{3}-\lambda_{4}$ ), where $\lambda_{1,2,3,4}$ are the square roots of the eigenvalues of $\rho_{12}\left[\left(\sigma^{y} \otimes \sigma^{y}\right) \rho_{12}^{*}\left(\sigma^{y} \otimes \sigma^{y}\right)\right]$ in a nonincreasing order [33-35]. Figure 4(a) shows that concurrence is also tunable by the gate voltage (dashed lines) and follows a similar trend to that of magnon entanglement (solid lines). Representative density states for entangled and separated two-qubit states are shown in Fig. 4(b). Here, there is a threshold entanglement of magnons near 0.082, above which the magnonic channel can mediate the entanglement of two qubits. To realize this proposal, one may optimize the material properties of $\mathrm{vdW}$ magnets and identify those with smaller exchange and anisotropy, such that they can readily be integrated with mature qubit platforms, including nitrogen-vacancy centers and superconducting qubits $[36,37]$. As an alternative, one may find quantum platforms working at the strong-field regime, which have already been indicated in recent experiments $[38,39]$.

\section{DISCUSSION AND CONCLUSION}

Coupling and decoupling of two or more qubits in a desirable way is an important topic in quantum technologies, such as quantum manipulation and quantum computing. The switchable entanglement channel in van der Waals magnets provides a route to prepare the entangled states of two qubits and may further enable the tunability of entanglement by electric means, which may take advantage of the $2 \mathrm{D}$ magnets for quantum processing. For example, one may design quantum logic gates based on tunable qubit-qubit coupling, which will be a building block for quantum computing. On the other hand, the magnonic systems fall into the class of continuous-variable systems, and 
thus, magnon-magnon entanglement may find its application in continuous-variable quantum information, such as quantum teleportation and quantum key distribution [12], complementing widely studied mechanical oscillators.

In conclusion, we show that magnons in a layered vdW magnet are entangled and that this entanglement is subject to a sudden death before the magnetic transition field. The essential physics is ascertained to be the competing influence of parametric interactions of the two types of magnons simultaneously and coherent transfer between the magnon states. On the practical side, our observation enables both magnetic and electrical means to switch the entanglement channel on and off and further makes it possible to utilize such a channel to bridge the entanglement of two or more qubits. These features are absent in the normal crystalline magnet because of the smallness of anisotropy compared with the exchange field and limited tunability range of the magnetic parameters by electric fields.

\section{ACKNOWLEDGMENTS}

H.Y.Y acknowledges the European Union's Horizon 2020 research and innovation programme under Marie Skłodowska-Curie Grant Agreement SPINCAT No. 101018193. R.A.D. has received funding from the European Research Council (ERC) under the European Union's Horizon 2020 research and innovation programme (Grant No. 725509). A.K. is funded by the Research Council of Norway through its Centers of Excellence funding scheme (Project No. 262633 “QuSpin”).

[1] C. Gong, L. Li, H. Ji, A. Stern, Y. Xia, T. Cao, W. Bao, C. Wang, Y. Wang, and Z. Q. Qiu, et al., Discovery of intrinsic ferromagnetism in two-dimensional van der Waals crystals, Nature 546, 265 (2017).

[2] B. Huang, G. Clark, E. Navarro-Moratalla, D. R. Klein, R. Cheng, K. L. Seyler, D. Zhong, E. Schmidgall, M. A. McGuire, and D. H. Cobden, et al., Layer-dependent ferromagnetism in a van der Waals crystal down to the monolayer limit, Nature 270, 546 (2017).

[3] K. S. Burch, D. Mandrus, and J.-G. Park, Magnetism in two-dimensional van der Waals materials, Nature 563, 47 (2018).

[4] M. Gibertini, M. Koperski, A. F. Morpurgo, and K. S. Novoselov, Magnetic 2D materials and heterostructures, Nat. Nanotech. 14, 408 (2019).

[5] X. Wang, J. Tang, X. Xia, C. He, J. Zhang, Y. Liu, C. Wan, C. Fang, C. Guo, and W. Yang, et al., Current-driven magnetization switching in a van der Waals ferromagnet $\mathrm{Fe}_{3} \mathrm{GeTe}_{2}$, Sci. Adv. 5, eaaw8904 (2019).

[6] K. Dolui, M. D. Petrovic, K. Zoliner, P. Plechac, J. Fabian, and B. K. Nikolic, Proximity spin-orbit torque on a twodimensional magnet within van der Waals heterostructure: Current-driven antiferromagnet-to-ferromanget reversible nonequilibrium phase transition in bilayer $\mathrm{CrI}_{3}$, Nano. Lett. 20, 4288 (2018).
[7] C. Wang, Y. Gao, H. Lv, X. Xu, and D. Xiao, Stacking Domain Wall Magnons in Twisted van der Waals Magnets, Phys. Rev. Lett. 125, 247201 (2020).

[8] W. Xing, L. Qiu, X. Wang, Y. Yao, Y. Ma, R. Cai, S. Jia, X. C. Xie, and W. Han, Magnon Transport in Quasi-TwoDimensional van der Waals Antiferromagnets, Phys. Rev. X 9, 011026 (2019).

[9] T. Liu, J. Peiro, D. K. de Wal, J. C. Leutenantsmeyer, M. H. D. Guimaraes, and B. J. van Wees, Spin caloritronics in a $\mathrm{CrBr}_{3}$-based magnetic van der Waals heterostructure, Phys. Rev. B 101, 205407 (2020).

[10] V. Gupta, T. M. Cham, G. M. Stiehl, A. Bose, J. A. Mittelstaedt, K. Kang, S. Jiang, K. F. Mak, J. Shan, R. A. Buhrman, and D. C. Ralph, Manipulation of the van der Waals magnet $\mathrm{Cr}_{2} \mathrm{Ge}_{2} \mathrm{Te}_{6}$ by spin-orbit torques, Nano. Lett. 20, 7482 (2020).

[11] X. Liu and M. C. Hersam, 2D materials for quantum information science, Nat. Rev. Mater. 4, 669 (2019).

[12] S. L. Braunstein and P. van Loock, Quantum information with continuous variables, Rev. Mod. Phys. 77, 513 (2005).

[13] M. Aspelmeyer, T. J. Kippenberg, and F. Marquardt, Cavity optomechanics, Rev. Mod. Phys. 86, 1391 (2014).

[14] H. Y. Yuan, S. Zheng, Z. Ficek, Q. Y. He, and M.-H. Yung, Enhancement of magnon-magnon entanglement inside a cavity, Phys. Rev. B 101, 014419 (2020).

[15] A. Kamra, E. Thingstad, G. Rastelli, R. A. Duine, A. Brataas, W. Belzig, and A. Sudbø, Antiferromagnetic magnons as highly squeezed Fock states underlying quantum correlations, Phys. Rev. B 100, 174407 (2019).

[16] D. M. F. Hartmann, J. J. Wouters, D. Schuricht, R. A. Duine, and A. Kamra, Intersublattice entanglement entropy as an extensive property in antiferromagnets, arXiv:2103.04809.

[17] J. Li and S.-Y. Zhu, Entangling two magnon modes via magnetostrictive interaction, New J. Phys. 21, 085001 (2019).

[18] Z. Zhang, M. O. Scully, and G. S. Agarwal, Quantum entanglement between two magnon modes via Kerr nonlinearity driven far from equilibrium, Phys. Rev. Res. 1, 023021 (2019).

[19] X. X. Zhang, L. Li, D. Weber, J. Goldberger, K. F. Mak, and J. Shan, Gate-tunable spin waves in antiferromagnetic atomic bilayers, Nat. Mater. 19, 838 (2020).

[20] H. Y. Yuan and Rembert A. Duine, Universal field dependence of magnetic resonance near zero frequency, Phys. Rev. B 103, 134440 (2021).

[21] T. Holstein and H. Primakoff, Field dependence of the intrinsic domain magnetization of a ferromagnet, Phys. Rev. 58, 1098 (1940).

[22] L.-M. Duan, G. Giedke, J. I. Cirac, and P. Zoller, Inseparability Criterion for Continuous Variable Systems, Phys. Rev. Lett. 84, 2722 (2000).

[23] R. Simon, Peres-Horodecki Separability Criterion for Continuous Variable Systems, Phys. Rev. Lett. 84, 2726 (2000).

[24] G. Adesso and F. Illuminati, Entanglement in continuous variable systems: Recent advances and current perspectives, J. Phys. A: Math. Theor. 40, 7821 (2007). 
[25] H. Y. Yuan, P. Yan, S. Zheng, Q. Y. He, K. Xia, and M.H. Yung, Steady Bell State Generation via Magnon-Photon Coupling, Phys. Rev. Lett. 124, 053602 (2020).

[26] Here, the vacuum state, $|00\rangle$, in the joint Fock space of sublattice magnons $\left(a_{1}\right.$ and $\left.a_{2}\right)$ is not the eigenstate of the Hamiltonian [Eq. (2)] due to the existence of parametric interactions in the system, and thus, it cannot be the real ground state. If we transform it into the dressed basis obtained by a squeezing operation on $a_{1}$ and $a_{2}$, the vacuum state of the squeezed magnons now becomes the ground state, which nevertheless consists of a high occupancy of sublattice magnons.

[27] D. Vitali, S. Gigan, A. Ferreira, H. R. Bohm, P. Tombesi, A. Guerreiro, V. Vedral, A. Zeilinger, and M. Aspelmeyer, Optomechanical Entanglement between a Movable Mirror and a Cavity Field, Phys. Rev. Lett. 98, 030405 (2007).

[28] As the dissipation increases to be comparable with the self-frequency $\left(\gamma \sim \omega_{0}\right)$, the magnitude of entanglement will decrease, while the critical field, $H_{c e}$, still exists. This regime is not considered in the current work.

[29] Y.-D. Wang and A. A. Clerk, Reservoir-Engineered Entanglement in Optomechanical Systems, Phys. Rev. Lett. 110, 253601 (2013).

[30] I. Bertelli, J. J. Carmiggelt, T. Yu, B. G. Simon, C. C. Pothoven, G. E. W. Bauer, Y. M. Blanter, J. Aarts, and T. van der Sar, Magnetic resonance imaging of spin-wave transport and interference in a magnetic insulator, Sci. Adv. 6, eabd3556 (2020).

[31] This value of coupling corresponds to the coupling strength of the magnet and qubit in the $\mathrm{MHz}$ regime, which is realized in experiments [30].
[32] In general, the spin waves of all wavevectors may mediate the coupling of two qubits. Here, we consider the low-temperature case, and thus, expect that the dominant contribution comes from the uniform-precession mode with zero wavevector.

[33] W. K. Wootters, Entanglement of Formation of an Arbitrary State of two Qubits, Phys. Rev. Lett. 80, 2245 (1998).

[34] H. Y. Yuan and M.-H. Yung, Thermodynamic entanglement of magnonic condensates, Phys. Rev. B 97, 060405(R) (2018).

[35] J. Zou, S. K. Kim, and Y. Tserkovnyak, Tuning entanglement by squeezing magnons in anisotropic magnets, Phys. Rev. B 101, 014416 (2020).

[36] G. Wendin, Quantum information processing with superconducting circuits: A review, Rep. Prog. Phys. 80, 106001 (2017).

[37] F. Casola, T. van der Sar, and A. Yacoby, Probing condensed matter physics with magnetometry based on nitrogen-vacancy centres in diamond, Nat. Rev. Mater. 3, 1 (2018).

[38] F. Luthi, T. Stavenga, O. W. Enzing, A. Bruno, C. Dickel, N. K. Langford, M. A. Rol, T. S. Jespersen, J. Nygård, P. Krogstrup, and L. DiCarlo, Evolution of Nanowire Transmon Qubits and Their Coherence in a Magnetic Field, Phys. Rev. Lett. 120, 100502 (2018).

[39] J. G. Kroll, W. Uilhoorn, K. L. van der Enden, D. de Jong, K. Watanabe, T. Taniguchi, S. Goswami, M. C. Cassidy, and L. P. Kouwenhoven, Magnetic field compatible circuit quantum electrodynamics with graphene Josephson junctions, Nat. Commun. 9, 4615 (2018). 